culture, and linguistics) (Fixsen, et. al., 2009; Becker, et al., 2020). Unique and hard-to-reach populations (UHRP) are defined based on physical location (i.e., remote or isolated), social position, or other vulnerabilities (i.e. member of an ethnic or racial minority group) (Thurman, \& Harrison, 2020). ORN classifies 26 types of UHRP these types are not mutually exclusive. A frequency analysis of the UHRP types was conducted. Bivariate correlations between UHRP types that had a minimum of 30 cases were performed. RESULTS/ANTICIPATED RESULTS: Among 746 TA requests selected, 226 had missing information about UHRP types and 29 had missing information TA levels. These requests were excluded from the frequency analysis. The three most common UHRP types were people living in rural or remote areas $(n=262,50 \%)$, people who are uninsured or underinsured $(n=162,31 \%)$, and people who inject drugs $(n=158,30 \%)$. Most TA requests were targeted (69\%), $23 \%$ were intensive, and $9 \%$ were basic. Bivariate correlations were performed between 21 UHRP types. Moderate (Pearson's $r=0.4-0.6$ ) or strong correlations $(r>0.6)$ were found for 11 occurrences for the UHRP type of 'LGBT', 8 for 'Mental Illness', and 7 for 'Veterans'. Strong correlations were found between 'Justice Involved' and 'Incarcerated' $(\mathrm{r}=0.645)$, and between 'Disabilities' and 'Chronic Pain' ( $r=0.603$ ). DISCUSSION/SIGNIFICANCE OF FINDINGS: There were more TA requests at targeted and intensive levels than basic levels suggesting the need for services to enhance readiness and build capacity. The moderate/strong correlations indicate that UHRP types were likely to coexist with other types. Future research can explore combining UHRP types that have moderate/ strong correlations.

Investigating the relationship between placement instability, mental health, behavioral and justice-related outcomes among sex-trafficked youth

Mekeila Cook ${ }^{1}$, Meagan Rainock ${ }^{2}$ and Breanna Thomas ${ }^{3}$ ${ }^{1}$ Meharry Medical College, ${ }^{2}$ Vanderbilt University and ${ }^{3}$ Indiana University

ABSTRACT IMPACT: This public health work contributes to the development and implementation of best practices for working with sex trafficked youth who experience placement instability and justice involvement. OBJECTIVES/GOALS: Youth removed from their home into foster care or a group home (i.e., placement instability) are vulnerable to sex trafficking. This study examines whether placement instability predicts mental health, behavioral and justicerelated outcomes among sex-trafficked girls. METHODS/STUDY POPULATION: Placement instability occurs when children are temporarily or permanently removed from their home and placed in foster care or a group home. Domestic minor sex trafficking is exploitation and abuse of children for commercial sexual purposes in exchange for money or other goods/services. We hypothesize that sex trafficked girls who experience placement instability will report more mental health challenges, substance use, abuse history and justice involvement than those without placement instability. Data came from participant files in a specialty court program from 2012-2014 ( $\mathrm{N}=184)$. Multiple sources contribute to the information contained court files; all data extracted by the research team come solely from the court files. Descriptive, bivariate, and logistic regression analyses were performed. RESULTS/ANTICIPATED RESULTS: All participants were (cis)female, $74 \%$ were African American, 96\% US citizens, with average age of 16 years. Threequarters of participants had a documented mental health challenge, such as depression and $88 \%$ reported substance use. Eighty-one percent of participants had been in a placement, with a group average of 4.5 placements. Girls with placement instability reported more mental health challenges $(\mathrm{p}<.001)$, substance use $(\mathrm{p}<.001)$, abuse $(\mathrm{p}<.001)$, running away $(\mathrm{p}<.001)$ and bench warrants $(\mathrm{p}<.001)$ than girls without placement instability. Logistic regression estimated housing instability was positively associated with mental health challenge, substance use, running away, number of bench warrants, and number of citations. DISCUSSION/SIGNIFICANCE OF FINDINGS: Among girls who have been trafficked, placement instability places them at greater risk for personal and behavioral challenges, including increased justice involvement. Comprehensive trauma-informed services should be provided to the family to help mitigate issues in the home.

41538

\section{Characterizing Opioid Overdose Hotspots for Targeted Overdose Prevention and Treatment}

Elizabeth A. Samuels, MD, MPH, MHS ${ }^{1}$, William Goedel, $\mathrm{PhD}^{2}$, Lauren Conkey, $\mathrm{MPH}^{3}$, Jennifer Koziol, $\mathrm{MPH}^{3}$, Sarah Karim ${ }^{3}$, Rachel P. Scagos, MPH $^{3}$, Lee Ann Jordison Keeler ${ }^{1}$, MSW, Rachel Yorlets ${ }^{2}$, $\mathrm{MPH}$, Neha Reddy ${ }^{4}$, MPH, Sara Becker, PhD², Roland Merchant ${ }^{5}$, MD $\mathrm{PhD}$ and Brandon D. L. Marshall ${ }^{2}, \mathrm{PhD}$

${ }^{1}$ Brown Emergency Medicine, ${ }^{2}$ Brown University School of Public Health, ${ }^{3}$ Rhode Island Department of Health, ${ }^{4}$ Warren Alpert Medical School of Brown University and ${ }^{5}$ Brigham and Women's Hospital

ABSTRACT IMPACT: Identifying factors associated with opioid overdoses will enable better resource allocation in communities most impacted by the overdose epidemic. OBJECTIVES/GOALS: Opioid overdoses often occur in hotspots identified by geographic and temporal trends. This study uses principles of community engaged research to identify neighborhood and community-level factors associated with opioid overdose within overdose hotspots which can be targets for novel intervention design. METHODS/STUDY POPULATION: We conducted an environmental scan in three overdose hotspots" two in an urban center and one in a small city" identified by the Rhode Island Department of Health as having the highest opioid overdose burden in Rhode Island. We engaged hotspot community stakeholders to identify neighborhood factors to map within each hotspot. Locations of addiction treatment, public transportation, harm reduction programs, public facilities (i.e., libraries, parks), first responders, and social services agencies were converted to latitude and longitude and mapped in ArcGIS. Using Esri Service Areas, we will evaluate the service areas of stationary services. We will overlay overdose events and use logistic regression identify neighborhood factors associated with overdose by comparing hotspot and non-hotspot neighborhoods. RESULTS/ANTICIPATED RESULTS: We anticipate that there will be differing neighborhood characteristics associated with overdose events in the densely populated urban area and those in the smaller city. The urban area hotspots will have overlapping social services, addiction treatment, and transportation service areas, while the small city will have fewer community resources without overlapping service areas and reduced public transportation access. We anticipate that overdoses will occur during times of the day when services are not available. Overall, overdose hotspots will be associated with increased census block level unemployment, homelessness, vacant housing, and low food security. DISCUSSION/SIGNIFICANCE OF FINDINGS: Identifying factors associated with opioid overdoses will enable better resource 